\title{
Decoupling Canopy Structure and Leaf Biochemistry: Testing the Utility of Directional Area Scattering Factor (DASF)
}

\author{
Jennifer Adams ${ }^{1, *}$, Philip Lewis ${ }^{2,3}$ and Mathias Disney ${ }^{2,3}$ (D) \\ 1 European Space Agency, ESA-ESRIN, Frascati 00044, Italy \\ 2 Department of Geography, University College London, Gower Street, London WC1E 6BT, UK; \\ p.lewis@ucl.ac.uk (P.L.); mathias.disney@ucl.ac.uk (M.D.) \\ 3 NERC National Centre for Earth Observation (NCEO), Leicester LE1 7RH, UK \\ * Correspondence: jennifer.adams@esa.int; Tel.: +39-069-418-0377
}

Received: 12 October 2018; Accepted: 26 November 2018; Published: 29 November 2018

\begin{abstract}
Biochemical properties retrieved from remote sensing data are crucial sources of information for many applications. However, leaf and canopy scattering processes must be accounted for to reliably estimate information on canopy biochemistry, carbon-cycle processes and energy exchange. A coupled leaf-canopy model based on spectral invariants theory has been proposed, that uses the so-called Directional Area Scattering Factor (DASF) to correct hyperspectral remote sensing data for canopy structural effects. In this study, the reliability of DASF to decouple canopy structure and biochemistry was empirically tested using simulated reflectance spectra modelled using a Monte Carlo Ray Tracing (MCRT) radiative transfer model. This approach allows all canopy and radiative properties to be specified a priori. Simulations were performed under idealised conditions of directional-hemispherical reflectance, isotropic Lambertian leaf reflectance and transmittance and sufficiently dense (high LAI) canopies with black soil where the impact of canopy background is negligible, and also departures from these conditions. It was shown that both DASF and total canopy scattering could be accurately extracted under idealised conditions using information from both the full 400-2500 nm spectral interval and the 710-790 nm interval alone, even given no prior knowledge of leaf optical properties. Departures from these idealised conditions: varying view geometry, bi-directional reflectance, LAI and soil effects, were tested. We demonstrate that total canopy scattering could be retrieved under conditions of varying view geometry and bi-directional reflectance, but LAI and soil effects were shown to reduce the accuracy with which the scattering can be modelled using the DASF approach. We show that canopy architecture, either homogeneous or heterogeneous 3D arrangements of canopy scattering elements, has important influences over DASF and consequently the accuracy of retrieval of total canopy scattering. Finally, although DASF and total canopy scattering could be retrieved to within $2.4 \%$ of the modelled total canopy scattering signal given no prior knowledge of leaf optical properties, spectral invariant parameters were not accurately retrieved from the simulated signal. This has important consequences since these parameters are quite widely used in canopy reflectance modelling and have the potential to help derive new, more accurate canopy biophysical information. Understanding and quantifying the limitations of the DASF approach as we have done here, is an important step in allowing the wider use of these methods for decoupling canopy structure and biochemistry.
\end{abstract}

Keywords: canopy biochemistry; hyperspectral reflectance; Directional Area Scattering Factor (DASF); spectral invariants; coupled leaf-canopy radiative transfer model; canopy scattering; Monte Carlo Ray Tracing (MCRT) 


\section{Introduction}

Terrestrial ecosystems influence the Earth's climate through various mass and energy exchange processes with the atmosphere [1]. Determining how much shortwave radiation is absorbed and reflected from vegetation canopies is vital to help understand and predict near-surface climate [2]. Earth Observation (EO) data are among the only means of providing measurements of solar radiation at the various spatial and temporal scales required for terrestrial-atmosphere interaction studies. The shortwave radiation budget describes how the fractions of absorbed or scattering radiation are a function of canopy optical properties and structure [3]. Therefore a remotely sensed signal carries information on the structural and biochemical characteristics of a canopy. The extraction of biochemical characteristics of vegetation canopies from remote sensing data is crucial for many studies, particularly carbon-cycle processes, foliar nitrogen and surface energy exchange [4]. However, canopy remote sensing data are subject to scattering at multiple levels, at the leaf and canopy scale, complicating the extraction of canopy biochemistry. This means the resulting remote sensing signal is a combination of coupled structural and biochemical signals. To retrieve biochemical information accurately and routinely from such data, these scattering processes must be accounted for $[4,5]$. The development of accurate canopy scattering models is therefore critical [3], and can enable the understanding of scattering processes exhibited by a canopy, and the factors that influence a remote sensing signal [6].

Thus far, very few canopy scattering models have been developed that incorporate scattering at both canopy and leaf scales. The LIBERTY (Leaf Incorporating Biochemistry Exhibiting Reflectance and Transmittance Yields) radiative transfer model (RTM) estimates optical properties of conifer needles including nitrogen, lignin and cellulose [7]; and LEAFMOD models internal leaf scattering [8]. However both models are limited in modelling leaf optical properties compared with the widely used PROSPECT leaf RTM [4]. More recently, PROSPECT has been integrated in to the SAIL canopy RTM (PROSAIL) to allow scattering at the leaf and canopy scales, however PROSAIL does not model explicitly the 3D structure of canopies, using simplified approximations of true 3D structural variation. Knyazikhin et al. [5] illustrated one of the first studies to thoroughly address combined leaf and canopy scattering, where the physically-based spectral invariant theory was applied to explicitly analyse the coupling of leaf biochemistry and canopy structure. In this approach, the concept of a Directional Area Scattering Factor (DASF) was conceived, that is able to correct hyperspectral reflectance data for canopy-structure effects and associated scattering processes. DASF is a structural term that can be directly retrieved from canopy reflectance spectra through physically based approaches that consider the fundamental laws of light interactions [5], and requires no prior knowledge or ancillary information on leaf optical properties. The concepts proposed are one of the only attempts at modelling spectral absorptions and scattering at both leaf and canopy scales [4], that has been neglected in previous studies of hyperspectral remote sensing of leaf biochemistry $[4,5,9]$.

Despite the recent integration of DASF into various applications (for example [2,10,11]), a rigorous empirical testing of the theory and its assumptions has yet to be achieved, partly due to the difficulty of measuring either canopy structural properties, or leaf and canopy scattering, in real canopies. Given the possible applications for estimating canopy biochemistry and the importance of such studies for climate modelling, estimating energy and gas exchanges, it is critical that the theory is well understood within its limits and assumptions. Here, we empirically test the approach made by Knyazikhin et al. [5], and the ability of DASF to correct bi-directional reflectance (BRF) data simulated using a Monte Carlo Ray Tracing (MCRT) RTM, for leaf and canopy scattering processes, and structural effects. In using a modelling approach, both spectral invariant parameters and canopy/leaf scattering can be benchmarked against 'reference' values that can be computed exactly in a modelling environment. Assumptions made throughout the conception of this theory are tested to examine the conditions under which DASF can correct for structural effects. Identifying such conditions, and rigorous testing of the effect of canopy and leaf scattering effects on BRF allows a more precise retrieval of leaf and canopy biochemistry, crucially, with quantified uncertainty. 


\section{Theory and Model Description}

\subsection{Spectral Invariants Theory}

Spectral invariant theory exploits simple algebraic combinations of leaf and canopy spectral reflectance and transmittance properties that become wavelength independent under certain assumptions, due to the size of scattering elements relative to the wavelengths of interest [12]. While scattering and absorption events are spectrally variant, canopy structure fundamentally controls interaction probabilities of photons. Thus spectrally invariant relationships can be developed to establish canopy structure parameters [13]. Solar radiation reflected by a vegetation canopy and measured by a sensor interacts with both green foliage and non-green material (branches, trunks). Spectral invariants considers green foliage matter only [5], where in a sufficiently dense vegetation canopy where the impact of the canopy background on total scattering is negligible, photon interactions with canopy elements can be described by leaf optical properties and canopy structure [5]. Radiation incident on an object can either be absorbed or scattered through reflectance, $\rho_{l}$, or transmittance, $\tau_{l}$ [14]. The fraction of radiation incident on a leaf that is scattered through reflectance or transmittance can be characterised by the leaf single scattering albedo, $\omega$. This fraction varies with wavelength, $\lambda$, and results from photon interactions at the leaf surface and the leaf interior:

$$
\omega_{\lambda}=i_{L} \omega_{\lambda}+s_{L}
$$

where $i_{L}$ is the fraction of radiation incident on the leaf that enters the leaf interior (i.e., leaf interceptance) and $s_{L}$ the fraction of radiation if reflected by the surface where $s_{L}=1-i_{L}$ and assumed to be wavelength independent. The transformed leaf albedo, $\omega_{\lambda}$ determines the fraction of radiation scattered from the leaf interior once it interacts with leaf biochemical constituents [14]. $\omega_{\lambda}$ varies with leaf biochemical constituents and leaf anatomical structure and is therefore independent from leaf surface properties.

However, canopy reflectance is also a function of structure, where photons that enter a canopy and encounter a leaf can be either absorbed or scattered. Scattered photons either hit another leaf or escape through gaps in the canopy, where under assumptions of energy conservation and a black soil, photons must eventually be absorbed or exit the canopy [13]. Under this assumption, Knyazikhin et al. [15] proposed the concept of a recollision probability, $p$, as a structural parameter and expresses the probability a photon scattered by a foliage element will interact with another foliage element [3]. Photons that do not recollide and escape through gaps between leaves have a probability of $1-p$. The directional gap density, $\rho(\Omega)$, uses the assertion that a gap can be defined as a point within the canopy that can be viewed outside in the viewing direction $\Omega$ [16]. $\rho(\Omega)$ is a function of both the amount of vegetation and its organisation (clumping and leaf orientation) within the canopy. Some photons that pass through the vegetation canopy may experience no interactions, where the remaining fraction are defined by the canopy interceptance, $i_{0}$. This property defines the portion of incident radiation that interacts with foliage elements for the first time [17], and depends on canopy structure and solar angle [5]. Accordingly, the three spectral invariant parameters $\left(i_{0}, p\right.$ and $\left.\rho(\Omega)\right)$ and leaf single scattering albedo $(\omega)$ can describe the BRF at a particular wavelength $\lambda$ and viewing angle $(\Omega)$ :

$$
B R F_{\lambda}(\Omega)=\frac{\rho(\Omega) i_{0}}{1-\omega_{\lambda} p} \omega_{\lambda}
$$

\subsection{Retrieval of DASF and Total Canopy Scattering (W)}

The three spectral invariant parameters can also be combined to determine the Directional Area Scattering Factor (DASF):

$$
D A S F=\rho(\Omega) \frac{i_{0}}{1-p}
$$


Huang et al. [12] show that $i_{0} /(1-p)$ is the mean number of interactions a photon will encounter before it exits the canopy assuming non-absorbing canopy elements (leaves). It is equivalent to the accumulated foliage area per unit ground area that an average photon trajectory will interact with over successive scattering orders, otherwise expressed as a Neumann series [16]. Accordingly, DASF estimates the ratio between the total one-sided leaf area (Leaf Area Index; LAI) and the canopy boundary leaf area seen in a given direction, thus is influenced by canopy structural properties such as the spatial distribution of trees, shape and size of trees, organisation of foliage within trees and the leaf surface properties.

In reality, canopies are not non-absorbing and a portion of incident radiation is absorbed by foliage elements. Absorption is spectrally dependent, therefore a large deviation between BRF and DASF occurs. However, in wavelengths that are weakly absorbing, multiple scattering dominates and is similar to radiative transfer in a non-absorbing canopy, for example in the Nir-Infrared (NIR) region (800-850 nm). If scattering photons are considered as radiation sources, the strength of this source can be determined by the leaf single scattering albedo, $\omega$. As a result, $p$ and DASF can be extracted given knowledge of $\omega$ in 2 or more weakly absorbing wavelengths. Considering that this information is currently not possible in EO data, Knyazikhin et al. [5] propose that DASF can be extracted from BRF in the 710-790 nm region, given no prior knowledge or ancillary information on leaf optical properties. In this region, scattering by leaf interior results mostly due to chlorophyll and dry matter spectra. Considering that dry matter exhibits little absorption and chlorophyll absorption declines throughout this spectral interval, leaf scattering is strong enough to dominate the BRF signal. Schull et al. [17] demonstrated reflectance in the 710-790 nm interval can be explained by a known leaf scattering spectra $\left(\omega_{0 \lambda}\right)$ and a spectrally invariant within-leaf recollision probability $\left(p_{L}\right)$ :

$$
\omega_{\lambda}=\frac{1-p_{l}}{1-p_{L} \omega_{0 \lambda}} \omega_{0 \lambda}
$$

Single scattering albedo can then be estimated considering that diffuse leaf albedo dominates:

$$
\omega_{\lambda} \approx i_{L} \omega_{\lambda}=\frac{1-p_{l}}{1-p_{L} \omega_{0 \lambda}} i_{L} \omega_{0 \lambda}
$$

where the leaf reference spectra is determined by absorption spectra of chlorophyll and dry matter. Substituting Equation (5) into Equation (2) can derive the BRF in a given wavelength $\lambda$ and a given viewing and illumination geometry $\Omega$ :

$$
\operatorname{BRF}_{\lambda}(\Omega)=\frac{i_{L} \rho(\Omega)\left(1-p_{L}\right) i_{0}}{1-p_{1} \omega_{0 \lambda}} \omega_{0 \lambda}
$$

where $p_{1}=p_{L}+i_{L} p\left(1-p_{L}\right)$. BRF can therefore be modelled using either $\omega$ and spectral invariants, or $\omega_{0 \lambda}$ and transformed spectral invariants which are normalised by $\omega_{0 \lambda}$. The ratio $B R F_{\lambda}(\Omega) / \omega_{0 \lambda}$ can obtain a linear relationship:

$$
\frac{B R F_{\lambda}(\Omega)}{\omega_{0 \lambda}}=p_{1} B R F_{\lambda}(\Omega)+i_{L} \rho(\Omega)\left(1-p_{L}\right) i_{0}
$$

DASF can be computed from the 710-790 nm with or without prior knowledge of the leaf single scattering albedo, and in vegetation canopies that are sufficiently dense that the canopy background impacts are negligible:

$$
D A S F=\rho(\Omega) \frac{i_{L} i_{0}}{1-p i_{L}}
$$

where Equation (2) can be rearranged so that BRF is expressed through:

$$
B R F_{\lambda}(\Omega)=\frac{\rho(\Omega) i_{0}}{1-\omega_{\lambda} p} \omega_{\lambda}=\frac{\rho(\Omega) i_{0} i_{L}}{1-p i_{L}}\left(\frac{1-p i_{L}}{1-\hat{\omega}_{\lambda} p i_{L}} \hat{\omega}_{\lambda}\right)=D A S F \cdot W_{\lambda}
$$


where $W$ is the total canopy scattering and $\hat{\omega}_{\lambda}=\omega_{\lambda} / i_{L}=\omega+s_{L}+i_{L} . W$ expresses the fraction of intercepted radiation scattered via reflectance or diffuse transmittance through the vegetation $[3,14]$ and is a function of both leaf surface and interior properties:

$$
W=\frac{1-p i_{L}}{1-\hat{\omega}_{\lambda} p i_{L}} \hat{\omega}_{\lambda}
$$

$W$ depends on the known leaf scattering albedo, and also on the absorption from multiple within-canopy photon interactions [5]. Normalising a BRF signal for structural effects characterised by the DASF can give an approximation of the total canopy scattering.

Lewis et al. [18] proposed an alternative method to express canopy reflectance using a Neumann series solution, in terms of parameters used to approximate reflectance, transmittance and scattering under energy conservation:

$$
\frac{c_{1} d_{1}}{1-p_{\infty} d_{1}}=\frac{c_{2} d_{2}}{1-p_{\infty} d_{2}}
$$

The parameters in Equation (11) can be represented in the following spectral invariant model that follows the Neumann series solution for multiple scattered components:

$$
p \propto \frac{a \omega}{1}-p \omega
$$

where $p$ represents the recollision probability and $a$ a geometric terms expressing the effect of interception and escape probabilities for first-order interactions. $\omega$ is the single scattering albedo defined as the combined scattering from leaf reflectance, $\rho_{l}$, and leaf transmittance, $\tau_{l}$ through $\omega=\rho_{l}+\tau_{l}$. The asymmetry of $\rho_{l}$ and $\tau_{l}$ is spectrally variant and influences canopy reflectance modelling, therefore equal asymmetry is often assumed for baseline experiments in spectral invariant studies. This model can be rearranged into the format of Equation (7) in a way that allows the parameters needed to derive DASF can be computed:

$$
\frac{\rho}{\omega}=a \rho+p
$$

\section{Model Simulations}

DASF and total canopy scattering $(W)$ were extracted under varying conditions that either corresponded to the assumptions presented in the previous sections, or departed from these assumptions. Hyperspectral BRF in the spectral region 400-2500 nm at $1 \mathrm{~nm}$ spectral resolution for each of the conditions was simulated using the librat Monte Carlo Ray Tracing (MCRT) radiative transfer model (RTM) [19]. The canopy radiative regime from librat is described using several inputs; a description of 3D canopy geometry (canopy scenes), leaf and soil scattering properties, illumination conditions and camera imaging characteristics. These inputs will be described below with respect to a baseline (or idealised) experiment and departures from the baseline experiment to test the ability of DASF to reconstruct $W$. The baseline experiment satisfies conditions of a dense structurally simple (homogeneous) canopy with underlying black soil, directional-hemispherical reflectance and a known leaf single scattering albedo with isotropic Lambertian leaf reflectance and transmittance with equal asymmetry. Further experiments describing non-idealised conditions were tested in terms of (a) varying solar geometry (bi-directional reflectance); (b) non-black soil; (c) heterogeneous canopies; (d) varying LAI and (e) unknown (or no prior) information on the $\omega$.

\subsection{Baseline Experiment}

The baseline experiment comprises a horizontally uniform canopy taken from the suite of abstract canopies in the RAdiation transfer Model Intercomparison (RAMI) fourth phase [20]. The abstract anisotropic homogeneous canopies consist of non-overlapping disc-shaped scatterers to represent canopy 
foliage elements. These discs are randomly distributed with a planophile Leaf Angle Distribution (LAD) within a slab-like volume, overlaid on a non-Lambertian horizontal plane (Figure 1a), designed to represent an anisotropically scattering underlying background. An LAI of 3 was chosen for the baseline experiment to ensure a dense canopy, corresponding the HOM25 experiment in RAMI-IV experiments [20].

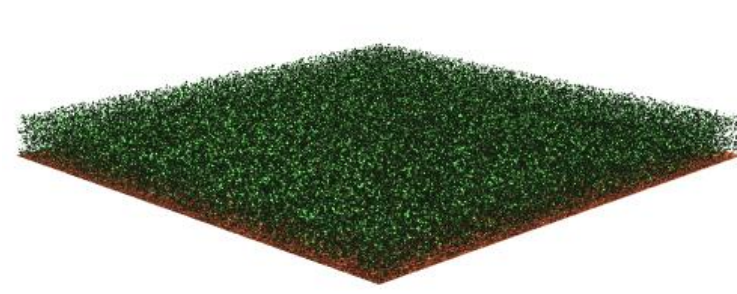

(a) HOM25 homogeneous canopy

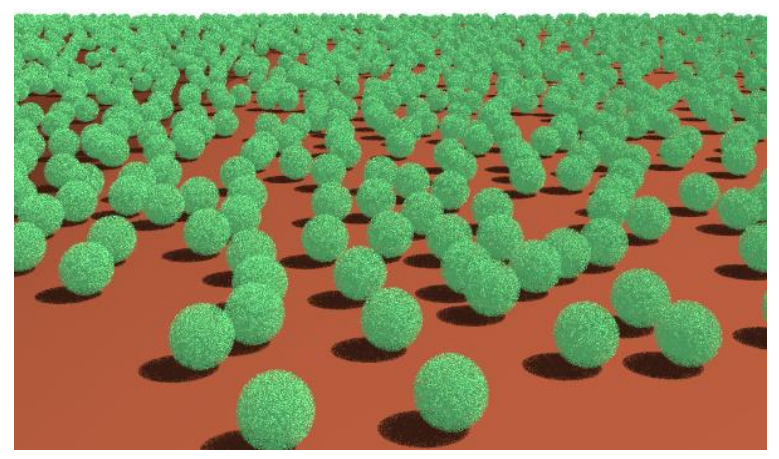

(b) HET12 heterogeneous canopy

Figure 1. RAMI-IV abstract canopies used in baseline experiments for homogeneous and heterogeneous canopies.

Leaf scattering properties were modelled using the PROSPECT leaf optical RTM [21], where input parameters were taken from the LOPEX dataset [22] as indicated in Table 1 for the known $\omega$ given prior knowledge of leaf optical properties. Leaf asymmetry was assumed to be equal leaf reflectance and leaf transmittance. Soil reflectance properties were defined under a totally absorbing black soil, given the assumption made by Knyazikhin et al. [5] that a dense canopy with dark soil should be used so that the impact of canopy background is negligible.

To satisfy conditions of directional-hemispherical reflectance, the diffuse-sky BRF output from librat, was used, defined as the cosine-weighted integral over a hemispherical illumination for a particular view angle. The directional component is varied through altering View Zenith Angle (VZA) and View Azimuth Angle (VAA). VZA is defined as the view angle measured away from the vertical and was varied from $0-75^{\circ}$ in steps of $15^{\circ}$ and VAA is kept constant at $0^{\circ}$.

Table 1. Absorption coefficient values for known and unknown $\omega$.

\begin{tabular}{ccc}
\hline & Known $\omega$ & Unknown $\omega$ \\
\hline Chlorophyll $\left(\mu \mathrm{g} / \mathrm{cm}^{2}\right)$ & 15.0 & 30.0 \\
Dry matter content $\left(\mathrm{g} / \mathrm{cm}^{2}\right)$ & 0.0053 & 0.0106 \\
Equivalent water thickness $(\mathrm{cm})$ & 0.0113 & 0.0226 \\
\hline
\end{tabular}

\subsection{Departures from Baseline Experiment}

To test whether DASF can be extracted without prior knowledge of $\omega$, Table 1 outlines an 'unknown' $\omega$ where the input parameters are doubled. In addition to the homogeneous canopy, two abstract anisotropic heterogeneous canopies were explored from RAMI-IV; HET12 and HET22, representing medium and high-density canopies respectively. Both are composed of identical non-overlapping spheres, comprised of similar disc- shaped scatterers as the homogeneous canopy (Figure 1b). The spheres are designed to represent individual plant crowns and are located over and only partially cover the same non-Lambertian horizontal plane as the previous homogeneous baseline canopy. Sphere radius' are set to $0.5 \mathrm{~m}$, with a sphere centre located at $0.51 \pm 0.0001 \mathrm{~m}$ above the plane, with random height distribution. The orientation of the normals of the discs follows a uniform (spherical) distribution function. 
In addition to the original homogeneous and heterogeneous canopies, the impact of LAI of extraction of $W$ was examined by adjusting the disc radius required to produce a specific LAI. For the homogeneous canopy, a specific LAI can be computed using $L A I=\pi r^{2} N / A$ where $N$ is the number of leaves, $A$ the canopy section area and $r$ the leaf radius. For the heterogeneous canopy, a specific LAI is calculated using $L A I=\pi r^{2} N / \pi r_{s}^{2}$ where $r_{s}$ represents the radius of sphere, set to $0.5 \mathrm{~m}$. $N$ is set to the same number of leaves for each original canopy, at 79,577 and 49,999 for the homogeneous and heterogeneous canopies respectively. LAI was varied from 1-10 in steps in 1.

To explore the impact of a non-dark underlying soil background, 6 soil spectra are chosen to represent different soil types with varying absorption components, and is demonstrated in Figure 2. One soil spectra was taken from Price's basic functions [23], representing a bright soil spectra, and a dry soil spectra from a test site in Eastern Germany was used, representing a loamy Chernozems soil dried in the laboratory. Four spectra were taken from the ASTER Version 2.0 Spectral Library to encompass a variety of different soil spectra, defined as black loam, brown clay, red silty-loam and white gypsum soils [24].

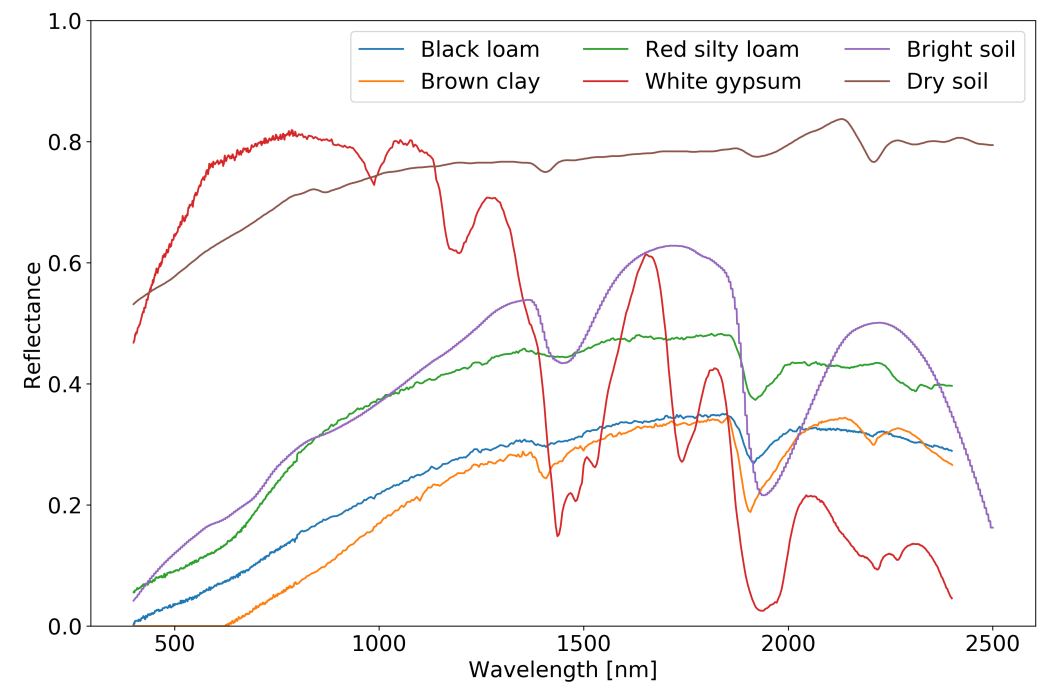

Figure 2. 6 soil spectra chosen to reflect varying absorption features taken from Baldridge et al. [24] and Price [23].

\subsection{Deriving DASF and $W$}

Following the theory outlined in Sections 2.1 and 2.2, DASF is extracted using the following method:

1. Calculate the leaf single scattering albedo $(\omega)$

2. Given a BRF simulated by librat:

(a) Plot values of $\omega$ against BRF using Lewis and Disney [14]'s method (Equation (13))

(b) Plot values of the ratio BRF/ $\omega$ vs. BRF to obtain a linear relationship using Knyazikhin et al. [5]'s method (Equation (7))

3. Determine parameters $a$ and $p$

4. Calculate DASF using the ratio $a /(1-p)$

5. Extract total canopy scattering $(W)$ by BRF/DASF

Spectral invariant parameters $a$ and $p$ were determined given BRF simulations via the two methods outlined above. DASF was derived using the full optical spectral interval 400-2500 nm and from the 710-790 nm region, based on the assumption that DASF can be extracted in a limited spectral interval. DASF in the 710-790 $\mathrm{nm}$ will furthermore be referred to as DASF ${ }_{710}$. In order to fully examine DASF, the parameters in which it depends on ( $a$ and $p$ ) need to be benchmarked against 
'reference' estimates. Given that $a$ is a geometric term that expresses the effect of interception and escape probabilities for first-order interactions, an explicit $a, a_{e}$, can be computed from the first-order scattering output $S_{1}$ and $\omega$ :

$$
a_{e}=\frac{S_{1}}{\omega}
$$

Interception and escape probabilities depend on leaf reflectance and transmittance. Therefore $a_{e}$ depends on the asymmetry of reflectance and transmittance, which vary spectrally. The recollision probability, $p$, can be applied separately for each order of interaction for each waveband, or over all interactions for each waveband. The latter definition refers to an 'effective' $p, p_{\text {eff }}$ which can be computed by inverted Equation (12):

$$
p_{\text {eff }}=\frac{1-\frac{a \omega}{\rho}}{\omega}
$$

$p_{\text {eff }}$ is expected to vary with wavelength due to different scattering and absorption processes that occur spectrally.

Both DASF and DASF 710 are used to correct BRF for canopy structural effects to estimate the total canopy scattering $W$ and $W_{710}$ respectively. To test the ability of DASF to reconstruct $W$, it is compared against a true $W, W_{T}$. This quantity is simulated concurrently with every librat simulation, where the plane that underlies each canopy scene is removed, therefore radiation that hits the non-existent plane immediately re-enters the scene. $W_{T}$ is simulated under diffuse sky conditions, and is normalised using a flat-white Lambertian panel. Discrepancy between $W$ and $W_{T}$ are documented by the the Sum of Squared Errors (SSE):

$$
S S E=\sum_{i=1}^{n}\left(y_{i}-\hat{y}_{i}\right)^{2}
$$

where $y_{i}$ represents the observed data $\left(W_{T}\right)$ and $\hat{y}_{i}$ the modelled data $\left(W\right.$ and $\left.W_{710}\right)$. Once $W$ is simulated, a canopy scattering $p$ can also be derived $\left(p_{w}\right)$ using:

$$
p_{w}=\frac{1-\frac{W}{\omega}}{1-W}
$$

\section{Results and Discussion}

Results are presented first for the baseline experiment for homogeneous and heterogeneous canopy architectures under conditions of theoretical assumptions and given that the leaf single scattering albedo is known. In the baseline experiment the viewing geometry is set to a VAA of $0^{\circ}$ and VZA of $45^{\circ}$. Results are then presented under the same conditions, however given an unknown leaf single scattering albedo. Finally, departures from the baseline experiment are presented.

\subsection{Baseline Experiment (Homogeneous): Known Single Scattering Albedo}

The performance of both methods to predict DASF are presented in Figure 3, where Lewis and Disney [14]'s method is presented in Figure 3a and Knyazikhin et al. [5]'s method in Figure 3b. For Lewis and Disney [14]'s method, the model fit to reflectance measurements can be found in as function of $\omega$. Model parameters are predicted at 0.24 and 0.59 for $a$ and $p$ respectively, which can therefore be used to calculate a DASF of 0.57. The second model of reflectance outlined in Knyazikhin et al. [5] was applied to the same canopy (Figure 3b). DASF was predicted the same, while $a$ was predicted at 0.25 in comparison to 0.24 for first model of reflectance, and $p$ was predicted at 0.56 in comparison to 0.59 .

Table 2 shows $a, p$, DASF and SSE values of $W$ for both homogeneous and heterogeneous canopies. For the HOM25 canopy in the 400-2500 nm spectral range with known single scattering albedo, Lewis and Disney [14]'s method results in an SSE value of 0.64 between $W$ and $W_{T}$. SSE computed in the same way for Knyazikhin et al. [5]'s method results in an SSE of 0.98; hence the first method is used from this point on to extract DASF. 


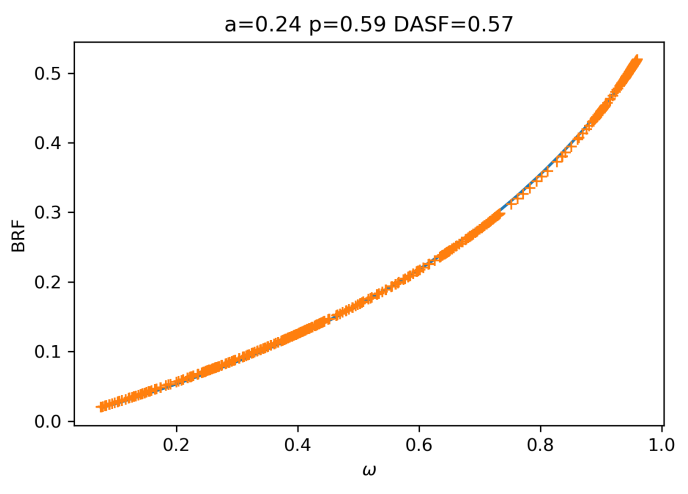

(a) Lewis and Disney [14]'s model

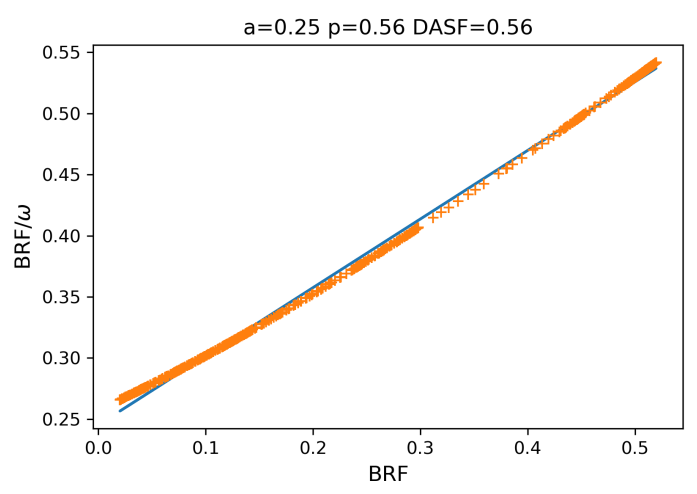

(b) Knyazikhin et al. [5]'s model

Figure 3. $a, p$ and DASF parameters extracted via both Lewis and Disney [14]'s and Knyazikhin et al. [5]'s models.

Table 2. Spectral invariants parameters $a$ and $p$, DASF and SSE for known and unknown $\omega$ using both full and 710-790 nm spectral region in HOM25 and HET12 canopies.

\begin{tabular}{|c|c|c|c|c|c|c|c|c|}
\hline & \multicolumn{4}{|c|}{ Homogeneous (HOM25) } & \multicolumn{4}{|c|}{ Heterogeneous (HET12) } \\
\hline & \multicolumn{2}{|c|}{$400-2500 \mathrm{~nm}$} & \multicolumn{2}{|c|}{$710-790 \mathrm{~nm}$} & \multicolumn{2}{|c|}{$400-2500 \mathrm{~nm}$} & \multicolumn{2}{|c|}{$710-790 \mathrm{~nm}$} \\
\hline & Known & Unknown & Known & Unknown & Known & Unknown & Known & Unknown \\
\hline $\mathbf{a}$ & 0.24 & 0.53 & 0.22 & 0.45 & 0.05 & 0.10 & 0.05 & 0.12 \\
\hline $\mathbf{p}$ & 0.59 & 0.04 & 0.62 & 0.21 & 0.55 & 0.07 & 0.54 & -0.08 \\
\hline DASF & 0.57 & 0.56 & 0.58 & 0.57 & 0.11 & 0.11 & 0.11 & 0.11 \\
\hline SSE & 0.64 & 1.12 & 0.45 & 0.50 & 0.02 & 0.03 & 0.03 & 0.019 \\
\hline
\end{tabular}

DASF is then used to correct BRF for structural effects to predict total canopy scattering, $W$, based on Lewis and Disney [14]'s model. Figure 4a displays the spectra of $W$, which mimics the shape of $\omega$, but is consistently lower. During wavelengths of rapid change in reflectance, specifically 710-750 nm, the total canopy scattering is closer to $\omega$. Figure $4 \mathrm{~b}$ shows $W$ reconstructed using DASF $(W)$ and $\operatorname{DASF}_{710}\left(W_{710}\right)$ and $W_{T}$ with respect to wavelength. Under conditions of directional-hemispherical reflectance, equal leaf reflectance and transmittance and black soil for a sufficiently dense homogeneous canopy, both DASF and total canopy scattering $(W)$ can be extracted with an SSE of 0.64 in the full optical domain and an SSE of 0.45 in the 710-790 nm spectral region. Consequently, DASF computed from 710-790 $\mathrm{nm}$ interval is able to predict total canopy scattering with less error than in the full spectral domain.

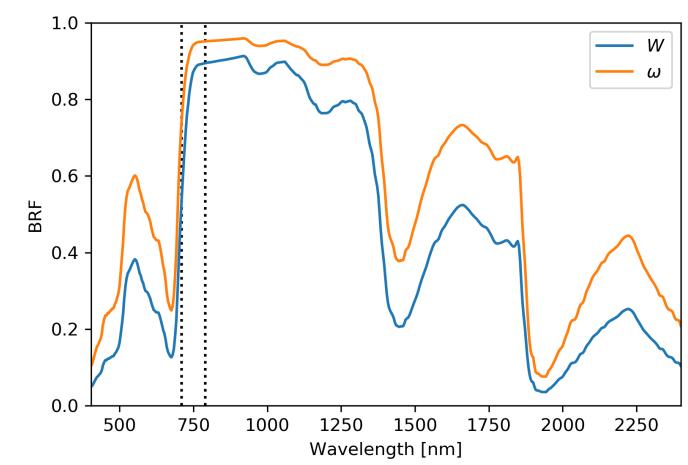

(a) Disparity of $W$ with $\omega$

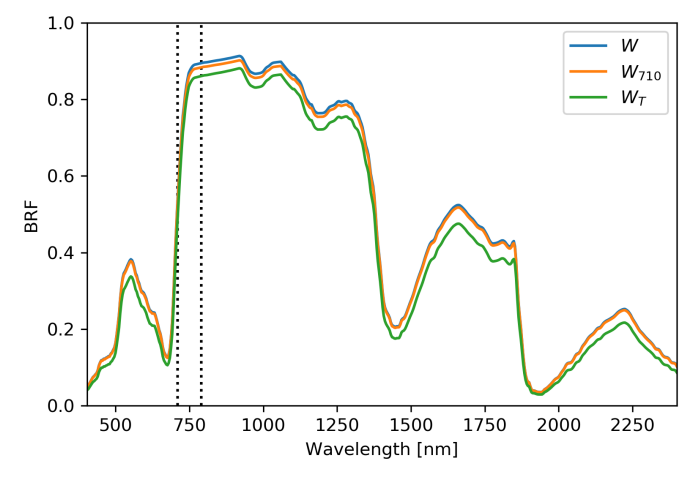

(b) Total canopy scattering $\left(W, W_{710}\right)$ and associated $W_{T}$

Figure 4. Comparison of $W$ and $\omega$, and prediction of total canopy scattering ( $W$ and $W_{710}$ ) compared to $W_{T}$, where dotted vertical lines represents $710-790 \mathrm{~nm}$ region used to compute $W_{710}$. 


\subsection{Baseline Experiment (Heterogeneous): Known Single Scattering Albedo}

Table 2 indicates that for the known $\omega$, values of DASF and SSE of $W$ for homogeneous and heterogeneous canopies confirms that canopy architecture has fundamental impacts on DASF. However, results show that expectations that homogeneous geometrically simple canopies should more reliably extract DASF and $W$ are proven to be incorrect, as heterogeneous canopies reconstruct $W$ more accurately. Predictions of $a$ and $p$ are considerably different for homogeneous and heterogeneous canopies. Canopy structure determines the escape probabilities for different scattering orders, and therefore $a$. In heterogeneous canopies, photons have a higher chance of escaping the sphere through different escape paths out of the sphere sides, whereas in a homogeneous canopy there are fewer escape paths [25], particularly in first-order interactions. The addition of more complicated canopy structure for heterogeneous canopies also slightly increases recollision probability. Mõttus et al. [25] found similar results, suggesting that canopies that are more structurally complex absorb radiation more efficiently. DASF values predicted for each canopy are also substantially different. The lower $a$ values demonstrated in the heterogeneous canopy are a result of increased escape probability from the spherical nature of the canopy, and the reduced ability to differentiate between first-order and multiple scattering for structurally more complex heterogeneous canopy. This, in combination with slightly higher $p$ (given that $D A S F=a /(1-p)$ ) results in a lower DASF for heterogeneous canopies. On the other hand homogeneous canopies are structurally simpler, therefore $a$ is more able to differentiate between first-order scattering and multiple scattering based on the interception and escape probabilities and is as a result higher. In combination with the lower $p$, DASF is therefore predicted higher in homogeneous canopies.

\subsection{Baseline Experiment: Unknown Single Scattering Albedo}

The parameters input into PROSPECT to simulate the unknown prior information on $\omega$ were double the proportions of chlorophyll, dry matter and water absorption parameters to the known $\omega$. $a, p$, DASF and SSE values are also shown in Table 2 for the 'Unknown' $\omega$ in both the full and limited spectral intervals. Despite inputting the unknown $\omega$, DASF and $W$ can still be extracted reliably with SSE as low as 0.019 for the HET12 canopy using information in the $710-790 \mathrm{~nm}$ range. This confirms the assumption that DASF and $W$ can be extracted given that no prior or ancillary knowledge of leaf single scattering albedo is known. Given that in reality the $\omega$ of a specific canopy is never known, the ability of this method to reconstruct $W$ is important. Additionally the discoveries that $W$ is more reliably extracted for heterogeneous canopies $\left(\mathrm{SSE}=0.019-0.03\right.$ ) and from $\mathrm{DASF}_{710}(\mathrm{SSE}=0.5)$ for homogeneous canopies is followed through when $\omega$ is unknown is used.

Further exploration into the model parameters demonstrates the tight coupling between canopy structure and leaf biochemistry, whereby inputting the unknown $\omega$ impacts the extraction of $a$ and $p$ parameters. However despite this, DASF can still be accurately predicted. Primarily, the unknown $\omega$ considerably affects the prediction of $p$, causing negative probabilities in some cases. This highlights that if $\omega$ is known, then $p$ can be determined. However in practice $\omega$ will not be known, and therefore $p$ cannot be interpreted using this method. On the other hand, if $p$ is known, $\omega$ can be derived, but if $p$ is not known, then $\omega$ cannot be derived [14]. This is important considering that $p$ is widely used parameter that is used to help determine LAI, vegetation type and other basic characteristics of vegetation canopies [26]. The incorrect prediction of $p$ raises questions to what structural effects have actually been accounted for.

\subsection{Departures from Baseline Experiment: VZA and SZA}

The dependence of $a, p$ and DASF on VZA and SZA are demonstrated in Figure 5. In changing the SZA, the VZA is set to a constant $45^{\circ}$, and in changing VZA, the SZA is set to $0^{\circ}$. Both $a$ and $p$ depend very weakly on SZA, causing DASF to vary a small amount with SZA. The distribution of points of first interaction is influenced by SZA, which therefore influences $a$. DASF shows different responses to 
SZA with homogeneous and heterogeneous canopies, where it is less stable in homogeneous canopies than heterogeneous canopies.
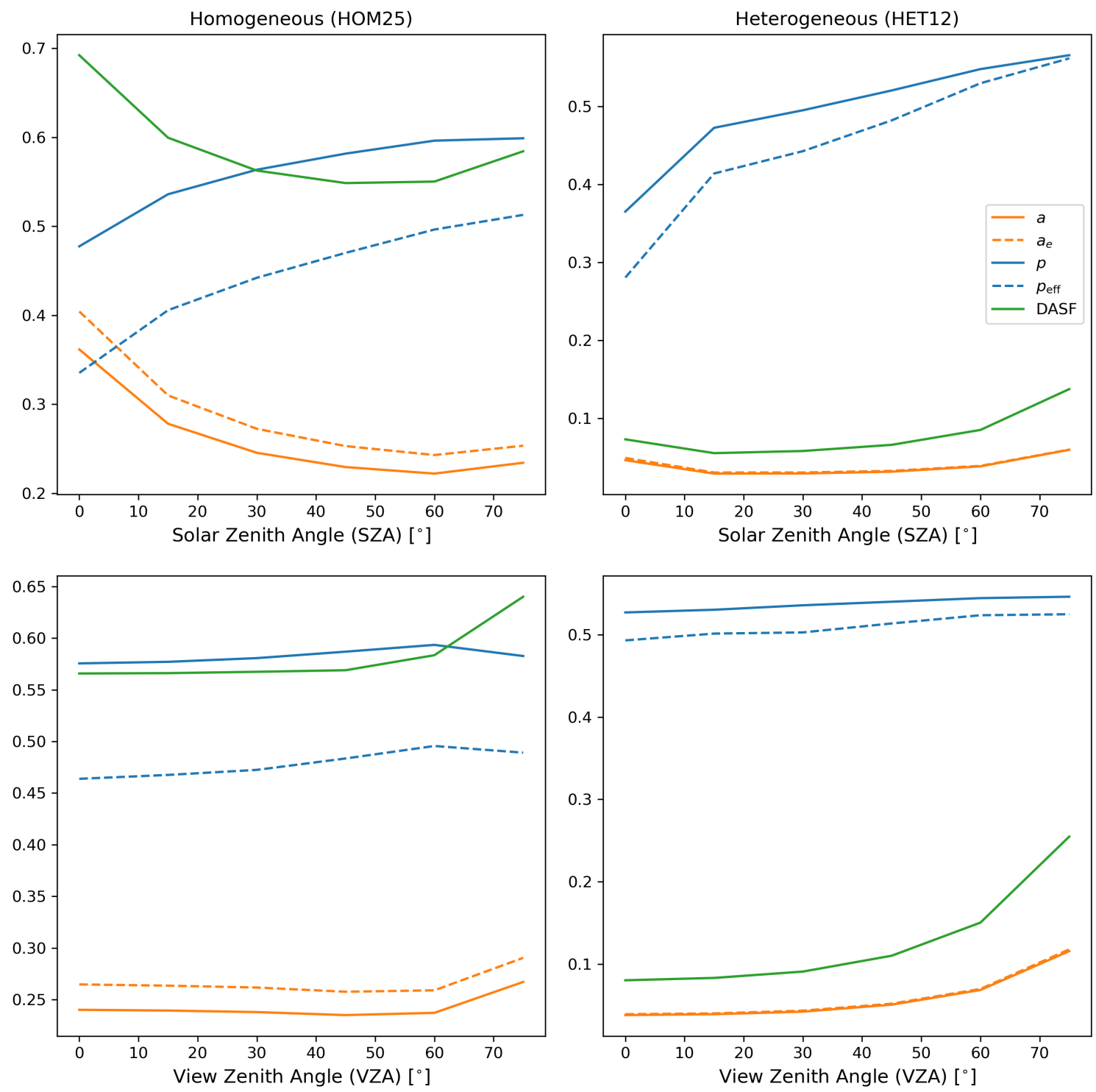

Figure 5. Dependence of $a, p$ and DASF with Solar Zenith Angle (SZA) (top panels) and View Zenith Angle (VZA) (bottom panels) for homogeneous (left panels) and heterogeneous (right panels) canopies.

VZA predominantly influences $a$, whereas $p$ depends weakly on VZA. Smolander and Stenberg [3] demonstrated that while $p$ depends primarily on scattering phase function and extinction, their results showed that $p$ depends weakly on the viewing geometry, which is confirmed in this study. $a$ is dependent on escape and interaction probabilities of the first order interaction, where the viewing geometry will affect the escape probability as view geometry impacts the distribution of points of the first interaction. Additionally, the escape probability is defined as the probability that a photon will escape in a given direction, thus the viewing direction affects the sensor's ability to detect escaped photons and will affect $a$, which is observed in both homogeneous and heterogeneous canopies. Despite this, DASF stays constant with VZA until $50^{\circ}$ in the homogeneous canopy, but increases with VZA in the heterogeneous canopy.

Figure 5 also highlights that recollision probability is consistently overestimated. For a homogeneous canopy, it was shown that while $W$ can be predicted accurately in comparison to $W_{T}$ (Table 2 SSE values), it is consistently overestimated. Using the method used to construct $W$ it follows that 
an overestimation of $w$ can be caused by either overestimation of $p$ or underestimation of $a$. Given that $p$ has been found to overestimate in comparison to $p_{\text {eff }}$, this suggests that for a homogeneous canopy structure, $p$ is overestimated. The most likely explanation for this is due to the assumption that $p$ is constant with scattering order made throughout the spectral invariant approach. This is not the case for a heterogeneous canopy, whereby $W$ can be predicted with SSE values of 0.02 and 0.03 (Table 2) for the full optical and 710-790 nm spectral intervals respectively. The similarity between model parameters and 'reference' parameters (Table 2) presents a reason why $W$ is likely reconstructed with small error.

\subsection{Departures from Baseline Experiment: $L A I$}

Figure 6 shows the effect of increasing LAI on $a, p$ and DASF for homogeneous and heterogeneous canopies using the full spectral domain (400-2500 nm) and 710-790 nm interval. The diffuse-sky $\mathrm{BRF}$ is used, where VZA is set to $45^{\circ}$. In comparison to results found by Lewis and Disney [14] and Smolander and Stenberg [3], $p$ saturation occurs much lower in this study for a homogeneous canopy, at 0.65 . Mõttus et al. [25] showed that recollision probability saturated at $\sim 0.79$ for heterogeneous canopies, demonstrating that saturation in this study also occurs lower at 0.73. Mõttus et al. [25] also showed that while homogeneous canopies resulted in lower recollision probabilities, the saturation of homogeneous and heterogeneous canopies is the same. This has most likely not occurred in this study due to the method of generating LAI scenes through increasing disc radius size, whereby in the homogeneous canopy this creates a 'big leaf' effect, which treats the canopy structure as a single big leaf rather than multiple small leaves as represented by the HOM25 canopy and real-world canopies. With increasing LAI $W$ departs from $W_{T}$, as demonstrated by SSE values presented in Table 3 . The effect of canopy LAI is significant when using spectrally invariant models of canopy reflectance, specifically on the recollision probability. Considering that canopy LAI scenes were generated through varying disc radius size and assuming the same sphere radius, a consequently stayed fairly stable with LAI, suggesting that the influence of canopy architecture is important. However, recollision probability is inherently coupled with LAI.

Considering that $\omega$ describes leaf scattering, and $W$ describes canopy scattering, discrepancies between $W$ and $\omega$ result from absorption from within-canopy photon multiple interactions. Kobayashi et al. [27] demonstrate that canopy scattering peaks at LAIs 1-2, then decreases with increasing LAI, which is supported here. Increasing the leaf area increases the probability of photon interaction with canopy elements until saturation begins to occur at higher LAIs. For LAIs of 1-2, the increases in recollision probability result in a scattering peak, therefore $W$ appears to interpret $\omega$ rather than canopy scattering. From an LAI of 3, increasing foliage elements causes absorption from within-canopy photon multiple interactions to dominate rather than scattering, and therefore canopy scattering departs from $\omega$. The saturation of $p$ with LAI is well documented in spectral invariant theory for high LAI canopies [3], and is likely an additional cause behind the increasing SSE values between $W$ and $W_{T}$ with increasing LAI values above 3 . Assuming that $p$ is constant with scattering order has been shown to work with little error [12], however this is no longer 'actual' $p$ which varies with scattering order and as a result can only be explained by the principle eigenvalue of the radiative transfer equation for low LAIs [28]. As aforementioned, the saturation of $p$ is particularly low due to the methodology used to vary LAI. Table 3 also highlights that given any LAI, DASF calculated from the 710-790 nm spectral region results in more accurate extraction of $W$, which confirms the ascertain made by [5] that information from the 710-790 $\mathrm{nm}$ region alone can be used to correct optical hyperspectral reflectance data for structural effects. 

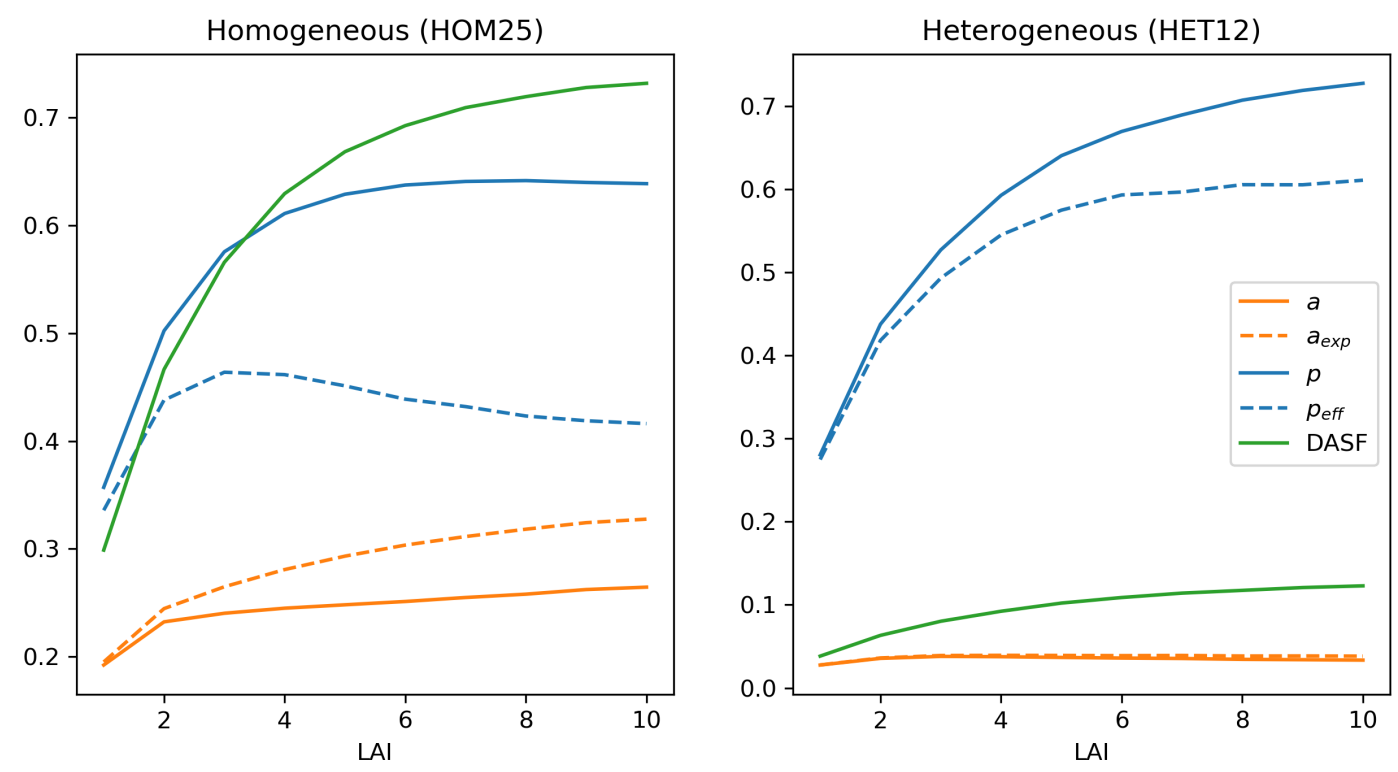

Figure 6. Dependence of $a, p$ and DASF with LAI for for homogeneous (left panel) and heterogeneous (right panel) canopies.

Table 3. SSE values between $W$ and $W_{710}$ for LAI 1-10 in homogeneous (HOM25) and heterogeneous (HET12) canopies.

\begin{tabular}{ccccc}
\hline & \multicolumn{2}{c}{ Homogeneous } & \multicolumn{2}{c}{ Heterogeneous } \\
\hline LAI & SSE $(\boldsymbol{W})$ & SSE $\left(\boldsymbol{W}_{\mathbf{7 1 0}}\right)$ & SSE $(\boldsymbol{W})$ & SSE $\left(\boldsymbol{W}_{\mathbf{7 1 0}}\right)$ \\
\hline 1 & 0.03 & 0.02 & 0.00 & 0.00 \\
2 & 0.25 & 0.19 & 0.01 & 0.01 \\
3 & 0.81 & 0.59 & 0.08 & 0.06 \\
4 & 1.74 & 1.21 & 0.15 & 0.11 \\
5 & 2.76 & 1.85 & 0.22 & 0.15 \\
6 & 3.86 & 2.53 & 0.39 & 0.25 \\
7 & 4.94 & 3.25 & 0.58 & 0.35 \\
8 & 5.73 & 3.74 & 0.82 & 0.49 \\
9 & 6.64 & 4.41 & 1.04 & 0.62 \\
10 & 7.15 & 4.76 & 1.28 & 0.75 \\
\hline
\end{tabular}

\subsection{Departures from Baseline Experiment: Soil}

The effect of soil scattering was investigated through simulating BRF in canopies with various underlying soils from the ASTER spectral library, and two soils from Price's basic functions [23]. Table 4 shows SSE values of $W$ with respect to $W_{T}$ for HOM25, HET12 and HET22 canopies for each soil and LAIs of 1,3,5 and 10. Based on SSE values, homogeneous canopies are more stable with soil scattering in comparison to heterogeneous canopies. As a result, the ability to reliably extract DASF and $W$ when soil scattering contaminates the reflectance signal depends on canopy type. For both canopies, there is a breakdown at an LAI of 1 when soil scattering is considered, which may be due to either a breakdown in the spectral invariant theory at LAI of 1 or that the low LAI could cause increased dominance of soil scattering spectra over canopy scattering therefore contaminate the reflectance signal. The SSE values also demonstrate that for optimum LAIs of 3 and $5, W$ is extracted more reliably, and as spectral invariant theory breaks down at higher LAIs, the ability to extract $W$ therefore decreases. Accordingly, 'trade-off' exists in terms of reliably extracting DASF with respect to soil and LAI. Increased LAI reduces the impact of soil scattering on the canopy and therefore improves the accuracy of reconstructing $W$. On the other hand higher LAI values in a canopy are associated with a breakdown in spectral invariant theory and therefore lower accuracies of $W$ reconstruction. 
Heterogeneous canopies are influenced considerably by soil scattering, due to the spatial distribution of trees. The breakdown of spectral invariant theory at high LAIs is demonstrated through investigations into the difference between medium and high density heterogeneous scenes. In high-density cases (HET22), the SSE values between $W$ and $W_{T}$ are higher at LAIs of 10 for black loam, brown to black clay and red organic-rich silty loam soils than for medium density cases (HET12). This suggests that the spectral invariant theory at high LAI and dense canopies contributes more to the breakdown rather than soil scattering properties. These soil experiments confirm assumptions that the theory of extracting DASF and $W$ works best under assumptions of black soil. While a sufficiently dense canopy is required, if the canopy is too dense or the soil scattering signal dominates, the $W$ cannot be extracted as reliably as it can under conditions of sufficiently dense vegetation with underlying black soil.

Table 4. SSE values for 6 different soil spectra for HOM25, HET12 (medium density) and HET22 (high density) canopies for LAI values 1, 3, 5 and 10.

\begin{tabular}{ccccccc}
\hline LAI & Black Loam & Brown Clay & Red Silty-Loam & White Gypsum & Bright & Dry \\
\hline HOM25 & & & & & & \\
\hline 1 & 5.88 & 7.68 & 5.51 & 6.42 & 7.68 & 4.39 \\
3 & 0.43 & 0.41 & 0.23 & 1.16 & 0.41 & 0.08 \\
5 & 1.97 & 1.86 & 1.69 & 0.57 & 1.86 & 0.99 \\
10 & 6.29 & 6.35 & 6.24 & 5.98 & 6.35 & 6.18 \\
\hline HET12 & & & & & & \\
\hline 1 & 150.42 & 92.74 & 115.08 & 18.90 & 92.74 & 115.86 \\
3 & 112.78 & 85.66 & 95.66 & 14.05 & 85.66 & 112.68 \\
5 & 108.22 & 90.88 & 97.45 & 14.82 & 90.88 & 123.57 \\
10 & 119.55 & 107.88 & 114.13 & 21.74 & 107.89 & 152.06 \\
\hline HET22 & & & & & & \\
\hline 1 & 84.55 & 114.00 & 69.02 & 12.16 & 62.36 & 77.99 \\
3 & 198.60 & 270.18 & 139.01 & 4.78 & 33.03 & 38.32 \\
5 & 212.22 & 283.41 & 151.55 & 3.22 & 28.17 & 33.49 \\
10 & 251.71 & 322.54 & 188.15 & 5.04 & 33.02 & 43.46 \\
\hline
\end{tabular}

\section{Conclusions}

Based on a rigorous empirical testing on RTM simulated hyperspectral BRF, DASF can reliably be extracted under conditions of directional-hemispherical reflectance, equal leaf asymmetry, sufficiently dense canopy and decorrelated soil and leaf spectra. Accordingly, DASF can be used to reliably correct hyperspectral reflectance data under idealised conditions for structural effects, to predict canopy scattering. Under the same conditions, the hypothesis that DASF could be extracted from the 710-790 nm spectral interval alone and used to correct for structural effects in the optical domain (400-2500 nm) was confirmed, often extracting canopy scattering more reliably than DASF extracted from the full optical domain. Secondly, the hypothesis that DASF could be extracted given that no prior or ancillary knowledge of leaf optical properties was also confirmed, through inputting the 'unknown' leaf single scattering albedo. This result is particularly important, as in reality, leaf optical properties of a canopy are not known. However, the study highlighted important consequences of no prior knowledge of leaf optical properties; if the leaf single scattering albedo is not known, the recollision probability cannot be found, and vice versa.

Varying viewing geometry and solar angle under assumptions of bi-directional reflectance were shown to influence the extraction of spectral invariant parameters and therefore DASF, particularly with respect to SZA. Canopy architecture was shown to have important influences over DASF and consequently the canopy scattering extracted. Notably it was shown that canopy scattering could be extracted more accurately in the more structurally complex heterogeneous scene. This method of 
interpreting reflectance to infer canopy scattering was shown to depend strongly on LAI, whereby with increasing LAI, canopy scattering was extracted progressively less accurately. It was hypothesised that this is due to the inability of recollision probability to describe increasing within-canopy absorption with increasing LAI as a result of saturation. Lastly, soil scattering effects caused a breakdown in the reliability with which total canopy scattering can be inferred. However, the contamination of soil scattering in reflectance data is evident, and filtering can be applied to remove these effects, allowing more reliable extraction of canopy scattering.

Canopy scattering due to canopy structure and leaf scattering as a result of leaf biochemistry are inherently coupled. Accordingly, in order to retrieve leaf biochemical information from hyperspectral remote sensing data, canopy scattering must be accounted for. This study demonstrated that DASF can reliably account for such canopy scattering effects under specific assumptions, and can thus be used to provide crucial leaf biochemistry estimates from remote sensing data which are essential to terrestrial ecosystem modelling.

Author Contributions: Formal analysis, J.A.; Methodology, J.A.; Supervision, M.D. and P.L.; Writing-original draft, J.A.; Writing-review and editing, M.D. and P.L.

Acknowledgments: M.D. and P.L. acknowledge NERC NCEO support. M.D. was supported in part by NERC Standard Grants NE/N00373X/1 and NE/P011780/1 and European Union's Horizon 2020 research and innovation programme under grant agreement No 640176 for the EU H2020 BACI project.

Conflicts of Interest: The authors declare no conflict of interest.

\section{References}

1. Ollinger, S.V.; Richardson, A.D.; Martin, M.E.; Hollinger, D.Y.; Frolking, S.E.; Reich, P.B.; Plourde, L.C.; Katul, G.G.; Munger, J.W.; Oren, R.; et al. Canopy nitrogen, carbon assimilation, and albedo in temperate and boreal forests: Functional relations and potential climate feedbacks. Proc. Natl. Acad. Sci. USA 2008, 105, 19336-19341. [CrossRef] [PubMed]

2. Wang, Y.; Buermann, W.; Stenberg, P.; Smolander, H.; Häme, T.; Tian, Y.; Hu, J.; Knyazikhin, Y.; Myneni, R.B. A new parameterization of canopy spectral response to incident solar radiation: Case study with hyperspectral data from pine dominant forest. Remote Sens. Environ. 2003, 85, 304-315. [CrossRef]

3. Smolander, S.; Stenberg, P. Simple parameterizations of the radiation budget of uniform broadleaved and coniferous canopies. Remote Sens. Environ. 2005, 94, 355-363. [CrossRef]

4. Ustin, S.L. Remote sensing of canopy chemistry. Proc. Natl. Acad. Sci. USA 2013, 110, 804-805. [CrossRef] [PubMed]

5. Knyazikhin, Y.; Schull, M.A.; Stenberg, P.; Mõttus, M.; Rautiainen, M.; Yang, Y.; Marshak, A.; Latorre Carmona, P.; Kaufmann, R.K.; Lewis, P.; et al. Hyperspectral remote sensing of foliar nitrogen content. Proc. Natl. Acad. Sci. USA 2013, 110, E185-E192. [CrossRef] [PubMed]

6. Huang, J.; Zeng, Y.; Kuusk, A.; Wu, B.; Dong, L.; Mao, K.; Chen, J. Inverting a forest canopy reflectance model to retrieve the overstorey and understorey leaf area index for forest stands. Int. J. Remote Sens. 2011, 32, 7591-7611. [CrossRef]

7. Dawson, T.; Curran, P.; Plummer, S. LIBERTY-Modeling the Effects of Leaf Biochemical Concentration on Reflectance Spectra. Remote Sens. Environ. 1998, 65, 50-60. [CrossRef]

8. Ganapol, B.D.; Johnson, L.F.; Hammer, P.D.; Hlavka, C.A.; Peterson, D.L. LEAFMOD: A New Within-Leaf Radiative Transfer Model. Remote Sens. Environ. 1998, 63, 182-193. [CrossRef]

9. Wang, Z.; Skidmore, A.K.; Wang, T.; Darvishzadeh, R.; Heiden, U.; Heurich, M.; Latifi, H.; Hearne, J. Canopy foliar nitrogen retrieved from airborne hyperspectral imagery by correcting for canopy structure effects. Int. J. Appl. Earth Obs. Geoinf. 2017, 54, 84-94. [CrossRef]

10. Yang, B.; Knyazikhin, Y.; Mõttus, M.; Rautiainen, M.; Stenberg, P.; Yan, L.; Chen, C.; Yan, K.; Choi, S.; Park, T; et al. Estimation of leaf area index and its sunlit portion from DSCOVR EPIC data: Theoretical basis. Remote Sens. Environ. 2017, 198, 69-84. [CrossRef] [PubMed]

11. Stenberg, P.; Manninen, T. The effect of clumping on canopy scattering and its directional properties: A model simulation using spectral invariants. Int. J. Remote Sens. 2015, 36, 5178-5191. [CrossRef] 
12. Huang, D.; Knyazikhin, Y.; Dickinson, R.E.; Rautiainen, M.; Stenberg, P.; Disney, M.; Lewis, P.; Cescatti, A.; Tian, Y.; Verhoef, W.; et al. Canopy spectral invariants for remote sensing and model applications. Remote Sens. Environ. 2007, 106, 106-122. [CrossRef]

13. Marshak, A.; Knyazikhin, Y.; Chiu, J.C.; Wiscombe, W.J. Spectrally Invariant Approximation within Atmospheric Radiative Transfer. J. Atmos. Sci. 2011, 68, 3094-3111. [CrossRef]

14. Lewis, P.; Disney, M. Spectral invariants and scattering across multiple scales from within-leaf to canopy. Remote Sens. Environ. 2007, 109, 196-206. [CrossRef]

15. Knyazikhin, Y.; Schull, M.A.; Xu, L.; Myneni, R.B.; Samanta, A. Canopy spectral invariants. Part 1: A new concept in remote sensing of vegetation. J. Quant. Spectrosc. Radiat. Transf. 2011, 112, 727-735. [CrossRef]

16. Stenberg, P. Simple analytical formula for calculating average photon recollision probability in vegetation canopies. Remote Sens. Environ. 2007, 109, 221-224. [CrossRef]

17. Schull, M.; Knyazikhin, Y.; Xu, L.; Samanta, A.; Carmona, P.; Lepine, L.; Jenkins, J.; Ganguly, S.; Myneni, R. Canopy spectral invariants, Part 2: Application to classification of forest types from hyperspectral data. J. Quant. Spectrosc. Radiat. Transf. 2011, 112, 736-750. [CrossRef]

18. Lewis, P.; Disney, M.; Knyazikhin, Y.; Quaife, T.; Schull, M. Modelling canopy reflectance with spectral invariants. In Proceedings of the 10th International Symposium on Physical Measurements and Signatures in Remote Sensing, Davos, Switzerland, 12-14 March 2007.

19. Lewis, P. Three-dimensional plant modelling for remote sensing simulation studies using the Botanical Plant Modelling System. Agronomie 1999, 19, 185-210.:19990302. [CrossRef]

20. Widlowski, J.-L.; Pinty, B.; Lopatka, M.; Atzberger, C.; Buzica, D.; Chelle, M.; Disney, M.; Gastellu-Etchegorry, J-P.; Gerboles, M.; Gobron, N.; et al. The fourth radiation transfer model intercomparison (RAMI-IV): Proficiency testing of canopy reflectance models with ISO-13528. J. Geophys. Res. Atmos. 2013, 118, 6869-6890. [CrossRef]

21. Jacquemoud, S.; Ustin, S.; Verdebout, J.; Schmuck, G.; Andreoli, G.; Hosgood, B. Estimating leaf biochemistry using the PROSPECT leaf optical properties model. Remote Sens. Environ. 1996, 56, 194-202. [CrossRef]

22. Hosgood, B.; Jacquemoud, S.; Andreoli, G.; Verdebout, J.; Pedrini, G.; Schmuck, G. Leaf Optical Properties Experiment 93 (LOPEX93); Report EUR-16095-EN; Joint Research Centre, Institute for Remote Sensing Applications: Ispra, Italy; European Commission: Luxembourg, 1995.

23. Price, J. On the information content of soil reflectance spectra. Remote Sens. Environ. 1990, 33, $113-121$. [CrossRef]

24. Baldridge, A.; Hook, S.; Grove, C.; Rivera, G. The ASTER spectral library version 2.0. Remote Sens. Environ. 2009, 113, 711-715. [CrossRef]

25. Mõttus, S.; Stenberg, P.; Rautiainen, M. Photon recollision probability in heterogeneous forest canopies: Compatibility with a hybrid GO model. J. Geophys. Res. Atmos. 2007, 112. [CrossRef]

26. Mõttus, M.; Stenberg, P. A simple parameterization of canopy reflectance using photon recollision probability. Remote Sens. Environ. 2008, 112, 1545-1551. [CrossRef]

27. Kobayashi, H.; Ryu, Y.; Baldocchi, D.D.; Welles, J.M.; Norman, J.M. On the correct estimation of gap fraction: How to remove scattered radiation in gap fraction measurements? Agric. For. Meteorol. 2013, 174-175, 170-183. [CrossRef]

28. Panferov, O.; Knyazikhin, Y.; Myneni, R.B.; Szarzynski, J.; Engwald, S.; Schnitzler, K.G.; Gravenhorst, G. The role of canopy structure in the spectral variation of transmission and absorption of solar radiation in vegetation canopies. IEEE Trans. Geosci. Remote Sens. 2001, 39, 241-253. [CrossRef]

(c) 2018 by the authors. Licensee MDPI, Basel, Switzerland. This article is an open access article distributed under the terms and conditions of the Creative Commons Attribution (CC BY) license (http://creativecommons.org/licenses/by/4.0/). 